\title{
1 Maternally-transferred thyroid hormones and life-history variation in birds
}

2 Bin-Yan Hsu ${ }^{1 *}$, Veli-Matti Pakanen², Winnie Boner ${ }^{3}$, Tapio Eeva ${ }^{1}$, Blandine Doligez ${ }^{4}$, Ton G.G.

3 Groothuis $^{5}$, Erkki Korpimäki ${ }^{1}$, Toni Laaksonen ${ }^{1}$, Asmoro Lelono $^{5}$, Pat Monaghan ${ }^{3}$, Tom Sarraude ${ }^{1,5}$,

4 Barbara Tschirren ${ }^{6}$, Robert L. Thomson ${ }^{7}$, Jere Tolvanen ${ }^{2}$, Rodrigo A. Vásquez ${ }^{8}$, Suvi Ruuskanen ${ }^{1}$

5

$6 \quad{ }^{1}$ Department of Biology, University of Turku, Finland

$7 \quad{ }^{2}$ Department of Ecology and Genetics, University of Oulu, Finland

$8{ }^{3}$ Institute of Biodiversity, Animal Healthy and Comparative Medicine, University of Glasgow,

9 Scotland, United Kingdom

$10{ }^{4}$ Department of Biometry and Evolutionary Biology, CNRS UMR 5558, Université de Lyon 1, France

$11{ }^{5}$ Groningen Institute for Evolutionary Life Sciences (GELIFES), University of Groningen, the

12 Netherlands

$13 \quad{ }^{6}$ Centre for Ecology and Conservation, University of Exeter, United Kingdom

$14{ }^{7}$ Fitzpatrick Institute of African Ornithology, DST-NRF Centre of Excellence, University of Cape Town,

15 South Africa

168 Instituto de Ecologíay Biodiversidad, Departamento de Ciencias Ecológicas, Facultad de Ciencias,

17 Universidad de Chile, Santiago, Chile

18

19

*Corresponding author: biyahs@utu.fi 


\section{Abstract}

Life-history traits vary largely across species and several physiological parameters have been proposed to be associated with life-history variation, such as metabolic rates, glucocorticoids, and oxidative stress. Interestingly, the association between thyroid hormones (THs) and life history variation has never been considered, despite a close interaction between THs and these physiological traits. Because of the crucial effects on embryonic development, THs can also induce transgenerational plasticity when transferred to developing offspring, for instance, via egg yolks in birds. In this study, we compiled a unique data set of maternal yolk THs in 34 bird species across 17 families and 6 orders, and tested for associations with various life-history traits. Our phylogenetic mixed models indicated that both concentrations and total amounts of the two most important forms of THs (T3 and T4) were higher in the eggs of migratory species than in those of resident species, and that there were higher total amounts of T3 in the eggs of precocial species than in those of altricial species. However, maternal THs did not show clear associations with any traits of the pace-of-life syndrome, such as developmental duration, growth rate, or lifespan. When taking environmental factors into account, we found that captive species deposited higher TH concentrations and larger amounts in the egg yolks than wild species. These findings suggest that maternal THs are likely involved in the evolution of life-history variation, or vice versa. comparative analysis, phenotypic plasticity 


\section{Introduction}

Life-history variation constitutes a large part of biodiversity. Across species, life-history traits are often inter-correlated and present along several continuums. For example, many life-history traits, such as lifespan, fecundity and growth rates, co-vary with one another and constitute a continuum representing the 'pace of life' (Ricklefs and Wikelski 2002; Dammhahn et al. 2018). On the one end of this continuum, species exhibit fast growth and development, early maturation, but also fast senescence and short lifespan ("fast" species). On the other end, species have slow growth and development, late maturation and first reproduction, but also live longer ("slow" species). Other lifehistory continuums, such as the nutrient source for developing embryos (from yolk or via placenta), income versus capital breeders, precocial to altricial developmental modes, and various degrees of migratory tendencies, also exist across various taxa in the animal kingdom.

Identifying the mechanisms underlying the evolution of life history differences has fascinated evolutionary biologists for decades. For example, metabolic rate has been proposed as a mediator for the pace-of-life (POL) continuum, as it exhibits a parallel pattern with fast species usually having higher basal and resting metabolic rates (BMR and RMR), while slow species having lower metabolic rates (e.g. Wikelski et al. 2003; Auer et al. 2018). Some studies also proposed other possible mediators, such as glucocorticoids (Hau et al. 2010), oxidative stress (Vágási et al. 2019), and blood glucose level (Tomasek et al. 2019). We propose that thyroid hormones (THs), which have regulatory functions on metabolism (Mullur et al. 2014) and interactions with glucocorticoids (Darras et al. 1996; Kulkami and Buchholz 2012; Cortés et al. 2014), may be an additional mechanism underlying life-history variation, such as the POL continuum, or explain previously observed associations between metabolic rate or circulating glucocorticoids and life-history variation. THs do not only regulate metabolisms in adults, but they are also transferred from mothers to developing offspring in all vertebrates (Ruuskanen and Hsu 2018). Such maternally-derived THs (hereafter "maternal THs") play a critical role for early embryonic development (Moog et al. 2017; Darras 2019). In humans, the levels of maternal THs are associated with new-born body mass and 
children's IQ (Medici et al. 2013; Korevaar et al. 2016). This suggests that variation in maternal THs can induce transgenerational phenotypic plasticity, which can constitute an important part of phenotypic variation in wild animals (Mousseau and Fox 1998; Moore et al. 2019). Nevertheless, the ecological and evolutionary significance of maternal THs in wild animals has been almost totally neglected (Ruuskanen and Hsu 2018). Recently, several experimental studies manipulating maternal

THs reported variable effects of yolk TH on nestling traits across species (Ruuskanen et al. 2016a;

Hsu et al. 2017, 2019a). These intriguing species differences suggest species might respond differently to elevated yolk TH levels and thus that maternal yolk THs might have co-evolved with life-history differences.

In this study, we explored the potential associations between maternal yolk TH levels and life-history strategies across 34 bird species. In birds, two maternally derived THs - triiodothyronine (T3) and thyroxine (T4) are transferred and stored in the egg yolk (Sechman and Bobek 1988; Prati et al. 1992). We asked whether the inter-specific variation of yolk THs would be associated with lifehistory traits across species, while accounting for environmental factors that may potentially contribute to the variation in yolk THs. The associations between maternal THs and life-history traits, if they exist, probably reflect the involvement of maternal THs in the evolution of life-history differences or vice versa.

Based on the known functions of THs, we considered three life-history continuums for which we hypothesized that maternal yolk THs may be associated with the related life-history traits. The first is the altricial-precocial developmental spectrum. At the two ends of this life-history continuum, precocial species and altricial species show distinctive ontogenetic patterns of visual and locomotory ability (Starck and Ricklefs 1998c), hypothalamic-pituitary-adrenal (HPA) axis (Wada 2008), hypothalamic-pituitary-thyroid (HPT) axis (McNabb 2006; de Groef et al. 2013) and thermoregulation (Price and Dzialowski 2018). In precocial species, the thyroid function already starts in the middle of incubation and the TH levels in thyroid gland and blood circulation both peak around hatching. By contrast, the thyroid function of altricial species starts days after hatching, and 
their circulating blood TH levels slowly increase towards the adult level (McNabb 2006; de Groef et al. 2013). Therefore, we hypothesized that such differences in the mode of development may be associated with yolk TH levels, either because higher maternal THs are required for a comparatively advanced development at hatching or because the developmental modes at least partly determine the transfer process of maternal THs, or both.

Second, we considered the life-history differences between migratory and resident species. THs have been known to regulate seasonal physiology and reproduction (Dardente et al. 2014; McNabb and Darras 2015; Nishiwaki-Ohkawa and Yoshimura 2016) and also moulting and feather regeneration (Vézina et al. 2009; Pérez et al. 2018). Several studies have also considered THs as part of the hormonal mechanism underlying migration (Pérez et al. 2016) and in general migratory species have higher BMR than resident species (Jetz et al. 2008). We thus hypothesized that maternal TH levels may be associated with a species' migratory status.

Third, we considered the slow-fast pace-of-life (POL) continuum (Ricklefs and Wikelski 2002; Dammhahn et al. 2018). As introduced above, metabolic rates (Wikelski et al. 2003; Auer et al. 2018), glucocorticoids (Hau et al. 2010), and oxidative stress (Vágási et al. 2018) have been proposed to mediate the POL-related life-history variation. Interestingly, THs appear to have associations with all of them. It is well known that THs have regulatory function on metabolism (Mullur et al. 2014), and in wild birds, within-species studies have confirmed positive correlations between blood THs and BMR and RMR (e.g. Chastel et al. 2003; Elliott et al 2013; Welcker et al. 2013). THs also have cross-talks with the HPA axis (Cortés et al. 2014) and may induce higher oxidative stress due to the metabolic effects (Villanueva et al. 2013). Therefore, it seems possible that THs may also be a mediator of pace of life.

In addition to life-history differences, some environmental factors may also contribute to the inter-specific variation of maternal yolk THs and should be taken into account. First, we expected that the foraging environment, through its direct influence on food composition, may influence the inter-specific variation of maternal yolk THs. For example, food conditions before and during the 
(Hsu et al. 2016). A possible explanation for this finding is the iodine content in the diet, as iodine is an essential ingredient for TH production (Bizhanova and Kopp 2009). In humans, even mild iodine deficiency during pregnancy has been shown to lead to deficiency of maternal T4 and hence detrimental effects on foetal development (Bath et al. 2013; Trumpff et al. 2013). In wild animals, environmental iodine availability is associated with antler weights of roe deer (Capreolus capreolus) via its influence on TH production (Lehoczki et al. 2011), and freshwater alligators (Alligator mississippiensis) have on average lower plasma T3 and T4 concentrations than their estuarine conspecifics (Boggs et al. 2011). Therefore, we hypothesized that iodine availability from the environment and diet (e.g. Eckhoff and Maage 1997) could contribute to part of the variation in maternal yolk THs. Moreover, captive environments differ in many aspects from wild environments, such as in food availability and safety from predators. Confinement and regular human disturbance might also pose as chronic stressors, which may influence THs (e.g. Angelier et al. 2016) and therefore maternal TH deposition. We therefore hypothesized that captivity may also influence maternal yolk THs. To test these hypotheses, we collected information on life-history traits pertaining to each hypothesis from the literature. The environmental variables were assigned for each species based on the sampled population. We measured maternal THs in egg yolks, constructing the first interspecific maternal TH data set. By combining data of life-history traits and maternal THs, we were able to run phylogenetic mixed-effects models using a Bayesian framework to test our hypotheses. 
Materials and Methods

Sample collection

We collected unincubated eggs from 34 species of birds across 17 families and 6 orders $(n=$

1-21 per species, median=7, Table S1). Nests of wild species were located by extensive nest searches

in the known breeding habitats of each species or from nest-box populations during 2016-2017. We

aimed at collecting eggs from species with varying body sizes and life-history traits and included

both precocial and altricial species. All eggs were collected before clutch completion (i.e. when

found, the eggs were cold and clutch was not completed) and one, randomly sampled egg per clutch

was collected. Therefore, in most of the cases, the laying order of the sampled egg was unknown.

Most of the wild species were sampled from Finnish populations near Turku $\left(60^{\circ} 27^{\prime} \mathrm{N}, 22^{\circ} 16^{\prime} \mathrm{E}\right)$, Oulu

$\left(65^{\circ} 01^{\prime} \mathrm{N}, 25^{\circ} 28^{\prime} \mathrm{E}\right)$ and Kauhava $\left(63^{\circ} 06^{\prime} \mathrm{N}, 23^{\circ} 04^{\prime} \mathrm{E}\right)$ regions, except thorn-tailed rayaditos

(Aphrastura spinicauda, sampled mostly from Chiloé island, Chile, $42^{\circ} 40^{\prime} \mathrm{S}, 73^{\circ} 59^{\prime} \mathrm{W}$ ) and collared

flycatchers (Ficedula albicollis, from Burgsvik, Gotland, Sweden, $57^{\circ} 02^{\prime} \mathrm{N}, 18^{\circ} 16^{\prime} \mathrm{E}$ ). The eggs of

captive homing pigeons (C. I. domesticus) and domesticated chicken (Gallus domesticus) and eggs of 

collected following all national and international guidelines.

$\underline{\text { Yolk TH analysis }}$

We extracted THs from the egg yolks and measured two THs (T3 and T4) at the University of

Turku and Turku Centre of Biotechnology. Yolk was separated from albumen after a short period of facilitate homogenizing.

We used the extraction protocol described previously (Ruuskanen et al. 2016b). In brief, we first added $2 \mathrm{ml}$ methanol to the yolk-MQ mixture (ca. $300 \mathrm{mg}$ ) and homogenized the sample. A known amount of ${ }^{13} \mathrm{C}_{12}$-T4 (Larodan) was added to each sample as an internal tracer, which allowed us to correct for extraction efficiency (i.e. recovery). Four $\mathrm{ml}$ of chloroform was then added and samples were centrifuged at $+4^{\circ} \mathrm{C} 1900 \mathrm{~g}$ for $15 \mathrm{~min}$. The supernatant was then collected and the pellet was re-extracted in 2:1 chloroform-methanol mixture, back-extracted with $0.05 \% \mathrm{CaCl}_{2}$ into aqueous phase, and re-extracted with chloroform:methanol:0.05\% $\mathrm{CaCl}_{2}$ mixture (3:49:48). The aqueous phase was further purified on Bio-Rad AG 1-X2 resin columns, eluted with $70 \%$ acetic acid, and then vacuum-evaporated overnight. Samples from different species were spread over extraction batches and the extraction batch ID was included as a random intercept in the statistical models.

We used a validated nano-flow liquid chromatography-mass spectrometry (LC-MS) protocol to measure yolk T3 and T4 simultaneously (Ruuskanen et al. 2018). In brief, the dry TH extracts were re-suspended in $150 \mu \mathrm{l} 0.1 \% \mathrm{NH}_{3}$ and further diluted with $0.01 \% \mathrm{NH}_{3}$. The dilution factor depended on the expected amount of T3 and T4 in each sample. Internal standards ${ }^{13} \mathrm{C}_{6}-\mathrm{T} 3$ and ${ }^{13} \mathrm{C}_{6}$-T4 were added to identify and also quantify T3 and T4 in the samples. A triple quadruple mass spectrometer 

using Thermo Xcalibur software (Thermo Fisher Scientific) and subsequently analysed by Skyline (MacLean et al. 2010). TH concentrations were calculated using the peak area ratios of sample to internal standard, corrected for recovery, and expressed as $\mathrm{pg} / \mathrm{mg}$ yolk. We further calculated the total amount of THs per yolk and expressed it as ng/yolk.

\section{Life-history traits and environmental factors}

\section{Altricial-Precocial continuum (i.e. developmental mode)}

We specified each species as precocial or altricial, following Starck and Ricklefs (1998a). The four species (the kestrel, Falco tinnunculus) were pooled with precocial or altricial species, respectively.

\section{Migratory status}

Each species was categorized as either migratory or resident, based on data compiled in Lehikoinen et al. (2003), McNab (2009) and Pap et al. (2015). When needed, the migratory status assignment was made based on the population from which we collected eggs.

III. Pace-of-life continuum

The pace-of-life continuum incorporates many related life-history traits, including a species' developmental duration, fecundity, metabolic rate, growth rate, and the rate of maturation and senescence.

(i) The data on developmental duration was collected mainly from the amniote life-history database compiled by Myhrvold et al. (2015) with supplementation from other literature (see supplementary materials). We collected the incubation duration and the time to 
period. We additionally calculated two more variables: the total length of development (incubation duration + time to fledging) and the proportion of prenatal development (incubation duration / total developmental length).

(ii) The growth rate data originated from Starck and Ricklefs (1998b) and was supplemented with other literature (see supplementary materials). For comparability, the logistic growth rate constants were used to represent the growth rate of each species. Whenever multiple sources of the growth rates are available for a species, an average was used.

(iii) Species' body mass, clutch size and number of clutches per year were obtained from Myhrvold et al. (2015).

(iv) Basal metabolic rates (BMR) were mainly obtained from McKechnie et al. (2006) and McNab (2009), supplemented by other literature (see supplementary materials).

(v) Maximum lifespan and ages at first reproduction originated from the database AnAge (Tacutu et al. 2013), except for the thorn-tailed rayaditos (Aphrastura spinicauda), for which the data was obtained from Moreno et al. (2005) and Quirici et al. (2019).

For environmental factors, we created two variables to represent a species' foraging environment

All life-history and environmental data we compiled for this study, the sources we consulted, and the final values used in all our models, can be found in the supplementary materials. 

corrected for body mass and phylogeny.

The four variables of developmental duration were strongly correlated with one another. phytools (Revell 2009, 2012). The first two PCs explained $99.74 \%$ of the variance (Table S3). The loadings suggested that PC1 is highly positively correlated with the time to fledging and the total each species to represent the developmental duration.

Because the data of life-history traits were not available for all species, we defined several pruned for the specific set of species where data was available. Each model set consists of separate models to analyse the concentrations and total contents of $\mathrm{T} 3$ and $\mathrm{T} 4$, respectively. This is because in oviparous species, the total contents of maternal hormones in the yolk is fixed after yolk formation. 
Therefore, the total content of hormones represents an initial maximum hormone availability and may represent different biological significance from the concentration. for 750000 iterations with a 350 thinning interval and burn-in of 50000, aiming for an approximately 2000 effective sample size for all parameters. Following Houslay and Wilson (2017), we used an uninformative parameter-expanded prior following a Cauchy distribution $(V=1, n u=1$, alpha.mu=0, alpha. $V=25^{\wedge} 2$ ) for all random factors. Tests using an uninformative inverse-Wishart prior or improper flat prior gave qualitatively same results, suggesting the results are robust against the choice of priors.

Each phylogeny set (Table 1) was correspondingly pruned and obtained from BirdTree.org (Jetz et al. backbone, 100 possible trees were generated and downloaded. All models were tested repeatedly across the 100 possible trees in each backbone and the posterior means of parameter estimates and their $95 \%$ credible intervals $(\mathrm{Cls})$ were stored. The average posterior mean and the average boundaries of the $95 \%$ Cls were calculated to account for phylogenetic uncertainty (Rubolini et al. 
and between the two backbones. Therefore we only reported the results based on the Hackett backbone.

As models across all possible phylogenetic trees produced highly similar results, we examined model performance by re-fitting all models with a consensus tree. The consensus tree was derived using the R package phytools (Revell 2012) for each phylogeny set. Model diagnostics were conducted by visual inspection on the trace plots for proper mixing and on autocorrelations. Model convergence was also checked using Gelman diagnostics provided in the R package coda (Plummer et al. 2006).

For all models, visual inspection did not raise any red flags for poor mixing or substantial autocorrelation and all models passed the Gelman diagnostics with all potential scale reduction factors $<1.05$.

Phylogenetic signals

$$
H^{2}=\frac{\sigma_{a}^{2}}{\sigma_{a}^{2}+\sigma_{s}^{2}+\sigma_{e}^{2}}
$$

where $\sigma_{a}^{2}$ represents the variance of the phylogeny; $\sigma_{s}^{2}$ represents the variance accounted by individual species; $\sigma_{e}^{2}$ represents the residual variance. The $H^{2}$ calculated this way is equivalent to the Pagel's $\lambda$ (Hadfield and Nakagawa 2010).

Because phylogenetic signal is in fact the proportion of the phylogenetic variance over the total phenotypic variance, the fixed factors included in the model will influence the value of the phylogenetic signal by changing the estimates of all variance components (Nakagawa and Schielzeth 2010). Therefore we calculated the phylogenetic signals for yolk T4 and T3 based on the phylogenetic mixed model that only included the life-history traits whose $95 \%$ Cls did not encompass 
bioRxiv preprint doi: https://doi.org/10.1101/775981; this version posted November 7,2019 . The copyright holder for this preprint (which was not certified by peer review) is the author/funder, who has granted bioRxiv a license to display the preprint in perpetuity. It is made available under aCC-BY-NC-ND 4.0 International license.

and body mass (see Results). In these models, all 34 species and the full phylogeny set was included

and we used a consensus tree from the Hackett backbone, derived by using the phytools package

(Revell 2012).

320

321 Table 1. Model, phylogeny and data sets defined in this study

\begin{tabular}{|c|c|c|c|c|}
\hline $\begin{array}{l}\text { Model } \\
\text { set }\end{array}$ & Phylogeny & Data set & $\begin{array}{l}\text { Model structure (fixed } \\
\text { factors) }\end{array}$ & Remark \\
\hline 1 & All 34 species & Full data set & $\begin{array}{l}\sim \text { developmental mode }+ \\
\text { migration }+ \text { body mass }+ \\
\text { maximum lifespan }+ \\
\text { foraging environment }+ \\
\text { captivity }\end{array}$ & \\
\hline $2 a$ & $\begin{array}{l}\text { Excluding } \\
\text { Coturnix japonica* }\end{array}$ & $\begin{array}{l}\text { Excluding } \\
\text { domesticated } \\
\text { Japanese quails } \\
\text { and domesticated } \\
\text { chicken }\end{array}$ & $\begin{array}{l}\sim \text { developmental mode }+ \\
\text { migration }+ \text { body mass }+ \\
\text { clutch size }+ \text { clutches per } \\
\text { year }+ \text { captivity }\end{array}$ & $\begin{array}{l}\text { Domesticated } \\
\text { quails and } \\
\text { chicken do not } \\
\text { lay clear } \\
\text { "clutches" of } \\
\text { eggs. }\end{array}$ \\
\hline $2 b$ & $\begin{array}{l}\text { Excluding } \\
\text { Coturnix japonica }\end{array}$ & $\begin{array}{l}\text { Excluding } \\
\text { Japanese quails }\end{array}$ & $\begin{array}{l}\sim \text { developmental mode }+ \\
\text { migration }+ \text { body mass }+ \\
\text { PC1 and PC2 of } \\
\text { developmental period }+ \\
\text { captivity }\end{array}$ & $\begin{array}{l}\text { Time to fledging } \\
\text { data was } \\
\text { missing for } \\
\text { Japanese quails }\end{array}$ \\
\hline 3 & $\begin{array}{l}\text { Excluding Gallus } \\
\text { gallus }\end{array}$ & $\begin{array}{l}\text { Excluding red } \\
\text { jungle fowl and } \\
\text { domesticated } \\
\text { chicken }\end{array}$ & $\begin{array}{l}\sim \text { developmental mode }+ \\
\text { migration }+ \text { body mass }+ \\
\text { age at first reproduction }+ \\
\text { captivity }\end{array}$ & $\begin{array}{l}\text { Age at first } \\
\text { reproduction } \\
\text { data was } \\
\text { missing for both } \\
\text { jungle fowl and } \\
\text { chicken }\end{array}$ \\
\hline 4 & $\begin{array}{l}28 \text { species with } \\
\text { BMR data }\end{array}$ & $\begin{array}{l}\text { Species with BMR } \\
\text { data }\end{array}$ & $\begin{array}{l}\sim \text { developmental mode }+ \\
\text { migration }+ \text { body mass }+ \\
\text { BMR + captivity }\end{array}$ & \\
\hline 5 & $\begin{array}{l}29 \text { species with } \\
\text { growth rate data }\end{array}$ & $\begin{array}{l}\text { Species with } \\
\text { growth rate data }\end{array}$ & $\begin{array}{l}\sim \text { developmental mode }+ \\
\text { migration }+ \text { body mass }+ \\
\text { growth rate }+ \text { captivity }\end{array}$ & \\
\hline
\end{tabular}

* In Model set 2a, both Japanese quails and domesticated chicken were removed from the "data 
Results

$\underline{\text { Inter-specific variation in yolk THs and phylogenetic signals }}$

We observed substantial inter-specific variation in both T3 and T4 concentrations (Fig. 1,

Table S1). Great tits (Parus major) and redshanks (Tringa totanus) had the lowest and highest yolk, $n=11$; redshank, mean $\pm S D=11.242 \pm 5.067 \mathrm{pg} / \mathrm{mg}$ yolk, $n=4$ ), and 18-fold for T4 (great tit, mean $\pm S D=0.989 \pm 0.292 \mathrm{pg} / \mathrm{mg}$ yolk, $\mathrm{n}=12 ;$ redshank, mean $\pm \mathrm{SD}=18.101 \pm 4.125 \mathrm{pg} / \mathrm{mg}$ yolk, $\mathrm{n}=$ 4). among species accounted for $60 \%-85 \%$ of the inter-specific variation in yolk THs (Table 2 ). On average, T3 has stronger phylogenetic signals than T4, although their 95\% Cls overlapped.

Table 2. Phylogenetic signals (i.e. phylogenetic heritability) for maternal yolk T3 and T4.

\begin{tabular}{clr}
\hline Hormone & Parameter & Posterior mean $[95 \% \mathrm{Cl}]$ \\
\hline T3 & concentration & $0.84[0.68,0.95]$ \\
& total content & $0.84[0.71,0.95]$ \\
\hline \multirow{2}{*}{ T4 } & concentration & $0.60[0.25,0.87]$ \\
& total content & $0.75[0.54,0.91]$ \\
\hline
\end{tabular}

Associations between yolk THs and life-history variables

The estimated posterior means and 95\% Cls from all models are presented in Fig. 2.

342 Precocial species deposited larger total amounts of THs in the egg yolks than altricial species (Fig. 3), but not higher TH concentrations. The model set 1 supported this difference with positive posterior means and $95 \% \mathrm{Cls}$, when controlling for species body mass and other covariates, especially for T3 contents (Fig. 2D). Also, migratory species generally deposited higher concentrations and also larger amounts of both THs in the egg yolks than resident species (Fig. 4), which were supported by the 
bioRxiv preprint doi: https://doi.org/10.1101/775981; this version posted November 7, 2019. The copyright holder for this preprint (which

was not certified by peer review) is the author/funder, who has granted bioRxiv a license to display the preprint in perpetuity. It is made available under aCC-BY-NC-ND 4.0 International license.

348 yolk TH concentration (Fig. 2). Along the slow-fast pace-of-life continuum, however, no traits were

349 found to be credibly associated with maternal yolk TH concentrations or contents (Fig. 2)

350

351 Associations between yolk THs and environmental variables

352 Although the raw data suggested that marine-bound species deposited larger amounts of yolk THs

353 than terrestrial species (Fig. S2), this was not supported by the phylogenetic mixed models (Fig. 2).

354 Between captive and wild species, credible differences in both yolk T3 and T4, and in both

355 concentrations and total contents (Fig. 2) were found. This suggested that captive species deposited

356 higher concentrations and larger amounts of yolk THs than wild species (Fig. 5). 
Discussion

Maternal THs and life-history variation

Among the three life-history continuums, our phylogenetic mixed models estimated

credible differences in maternal yolk THs between the migratory and resident life histories, and

between precocial and altricial developmental modes. However, contrary to our expectation, none

THs between precocial and altricial species, and between migratory and resident species still suggest that maternal THs are associated with the evolution of these two life-history continuums or vice versa.

THs and migration physiological transition before and after reproduction compared with migratory species. Therefore, migratory species may require a higher sensitivity or accuracy to fine-tune the transition. THs play a central role in the molecular mechanisms of photoperiodic response. The photoperiodic change influences the expression levels of the thyroid-stimulating hormones (TSH) in the hypothalamus, which subsequently induces reciprocal switching of two deiodinases (DIO2, converting T4 to active 
(Nakao et al. 2008; Dardente et al. 2014). This photoperiodic DIO2/DIO3 expression pattern further regulates the seasonal maturation of gonads. Interestingly, the photoperiodic DIO2/DIO3 gene expression pattern of great tits was only evident in a Swedish population but not in a southern German population (Perfito et al. 2012), in which the duration of the breeding seasons are likely to differ. Our result of higher yolk TH levels in migratory than resident species, not only in terms of similar pattern was not found in European starlings (Sturnus vulgaris, Bentley et al. 2013). Comparison of TH-related gene expression between conspecific populations that possess different migratory propensity, potentially in combination with TH manipulation, may help to identify the elements responsible for such differences.

Does precocial development require more maternal THs? mass), particularly T3, in the egg yolks than altricial species, which fits their distinct developmental amount of maternal THs for such an advanced ontogenetic trajectory in precocial birds. altricial development may be the placement of hatching on the ontogenetic timeline. Regardless of the developmental modes, the event of hatching requires a lot of physiological changes to prepare the chick for the "outside" world and therefore marks a major life-stage transition. As the altricial 
1998c; de Groef et al. 2013), one way to look at the shift is that the event of hatching is moved earlier along the ontogenetic timeline in the altricial development so that hatching occurs when the young is still poorly developed. Whether maternal THs mediate such a shift during the evolution from precocial to altricial development requires further studies, but according to our data such a change may coincide with a reduction in TH deposition over the evolutionary course. We therefore could expect intermediate levels of maternal THs in semi-precocial and semi-altricial species. Interestingly, the only semi-altricial species we measured, the Eurasian kestrel, indeed has higher maternal TH content than most other altricial species, but lower than most semi-precocial and precocial species (Fig. 3).

No size-independent associations between maternal THs and pace of life (POL)

Given the known function of THs on metabolism and interactions with glucocorticoids, it is surprising that we did not detect any associations between maternal THs and all tested POL-related traits (Fig. 2). Although yolk TH contents show strong correlations with species body mass, this is because large species produce large eggs and large yolks, hence higher total amounts of yolk THs. After controlling for body mass and phylogenetic relatedness, only clutch size showed a potential the egg yolks. Such a trend is probably driven by the difference between passerines and shorebirds (Fig. S3). Confirming such a relationship perhaps would require a larger sampling scheme that includes more species. our hypothesis that THs were mediators of POL. This result therefore implies that either (i) the effects of maternal THs may not be translated directly to blood THs or (ii) blood THs are not associated with POL-related traits. The former could be due to limited organizational effects of 
knowledge, there are only few experimental studies that have examined how maternal THs influenced blood THs and metabolic rates during the nestling phase. In the great tit, yolk TH elevation did not influence nestling RMR before fledging (Ruuskanen et al. 2016a). In the rock pigeon, yolk TH elevation raised the RMR in female nestlings but reduced it in males on hatching day, a pattern that was consistent with the effects on plasma T4 concentrations (but not T3) at day unexplained. Nevertheless, in domesticated chicken embryos the injection of THs into the yolk influenced the expression of two TH-transporters in the brain (van Herck et al. 2012). This suggests that differential exposure to maternal THs may influence brain TH availability. Such changes might have the potential to alter the sensitivity and responsiveness of the HPT axis in the long term. Therefore, the notion that maternal THs may organizationally shape the HPT axis cannot yet be ruled out. Another possibility to explain the lack of associations between maternal THs and POL-related traits is that blood THs are not associated with POL-related traits. This would be quite surprising considering the close associations between THs with metabolic rates, glucocorticoids, and oxidative stress. As POL-related traits are usually strongly associated with body mass, perhaps a much larger number of species is required to detect such potential associations after accounting for species body mass.

Above, we have discussed the potential role of maternal THs as mediators of life-history variation. However, it is possible that differences in life-histories, particularly in migratory behaviour and 457 developmental mode, may result in physiological differences that cause differential maternal TH deposition during egg formation across species. For example, migratory birds generally have higher BMR than residents (Jetz et al. 2008). Given the close relationship between BMR and THs, migratory species probably also have higher circulating blood TH levels. One study in two closely-related 
skylark species indeed found that the migratory Eurasian skylarks (Alauda arvensis) had higher prebreeding blood T3 levels than the resident Asian short-toed larks (Calandrella cheleensis, Zhao et al. 2017). A meta-analysis on the available blood TH data from the literature should be promising to test the associations between life-history variation and TH physiology but to our knowledge, such a meta-analysis has not yet been done. If in general migratory species keep comparatively higher mirroring the circulating TH levels in the system of migratory birds. differential yolk TH deposition may still reinforce the evolution of the life-history variation. Due to the critical role in embryonic development, maternal THs likely have short-term and long-term effects on offspring phenotype, such as nestling development, growth, and physiology (Ruuskanen et al. 2016a; Hsu et al. 2017, 2019a). If higher maternal THs would lend beneficial effects for a certain life-history strategy, and physiologically such a life history results in a higher maternal TH transfer, maternal THs may speed up the evolution of associated life-history traits (McGlothin and Galloway 2014). At the moment, this is a bold speculation. However, considering that yolk T3 deposition has a moderate heritability $\left(h^{2}=0.25\right.$, Ruuskanen et al. 2016c) and clear betweenindividual difference (Hsu et al. 2019b), there might be evolutionary potential for this to happen. Future studies may examine the temporal and quantitative differences in the expression patterns of TH-related genes in embryonic brains between migratory and resident species, and between precocial and altricial species, to know if there has been fundamental differences during the early developmental stage. Another angle would be examining whether birds hatching from eggs with elevated yolk THs may deposit higher yolk THs than control birds to test if maternal TH can induce any non-genetic inheritance. 
In terms of environmental factors, our models suggested differences in maternal yolk THs between captive and wild species, but not between terrestrial and marine-bound species.

Life in captivity is characterised by space confinement, (usually) unlimited food availability and a study, all the captive species have acclimated to captive lives for generations. Therefore the result that captive species deposit higher levels of THs in the egg yolks likely reflects some selection under captivity. The actual selective force is elusive at the moment, but probably related to traits like faster acclimation/habituation for anthropogenic diet or perhaps dampened stress response to confined space and constant human disturbance, which should be favoured in captivity.

The larger yolk TH contents in marine-bound species compared to terrestrial species, apparent from power to statistically partition the effects of environment and phylogeny because all sampled marine-bound species were from the order Charadriiformes. Future studies should therefore broaden the sampling scheme to overcome this problem. An alternative to test the relationship between foraging environment and maternal THs is to focus on a smaller clade, such as a single order or a single species inhabiting both inland and marine-bound populations. In the present study, the eggs of the little ringed plover (Charadrius dubius) were sampled from an inland population. The species, interestingly, had the lowest T4 content among all Charadriiform species, which implies 
captive model system or supplementing iodine-rich food to a wild population would be also an effective approach in this respect.

515 Conclusion

516 Our phylogenetic mixed models suggested that migratory species deposited higher levels of both

517 THs in the egg yolks than resident species, and precocial species deposited higher total content of T3

518 in the egg yolks than altricial species. In addition, captive species also consistently deposited higher

519 levels of yolk THs, suggesting some selection process under captivity. These identified patterns

520 suggest that maternal THs may be involved in the process of life-history evolution and can respond

521 to on-going selective forces. Further effort should be invested to uncover the underlying

522 physiological mechanisms.

\section{Acknowledgement}

525 We thank Jon Brommer for his kind and helpful statistical advice in the beginning stage of data

526 analysis. We thank Jorma Nurmi for excellent nest-finding skills and effort in the field. Mass

527 spectrometry analyses were performed at the Turku Proteomics Facility, University of Turku and Åbo

528 Akademi University. The facility is supported by Biocenter Finland. This study was funded by a grant from the Academy of Finland to SR (grant no. 286278). 


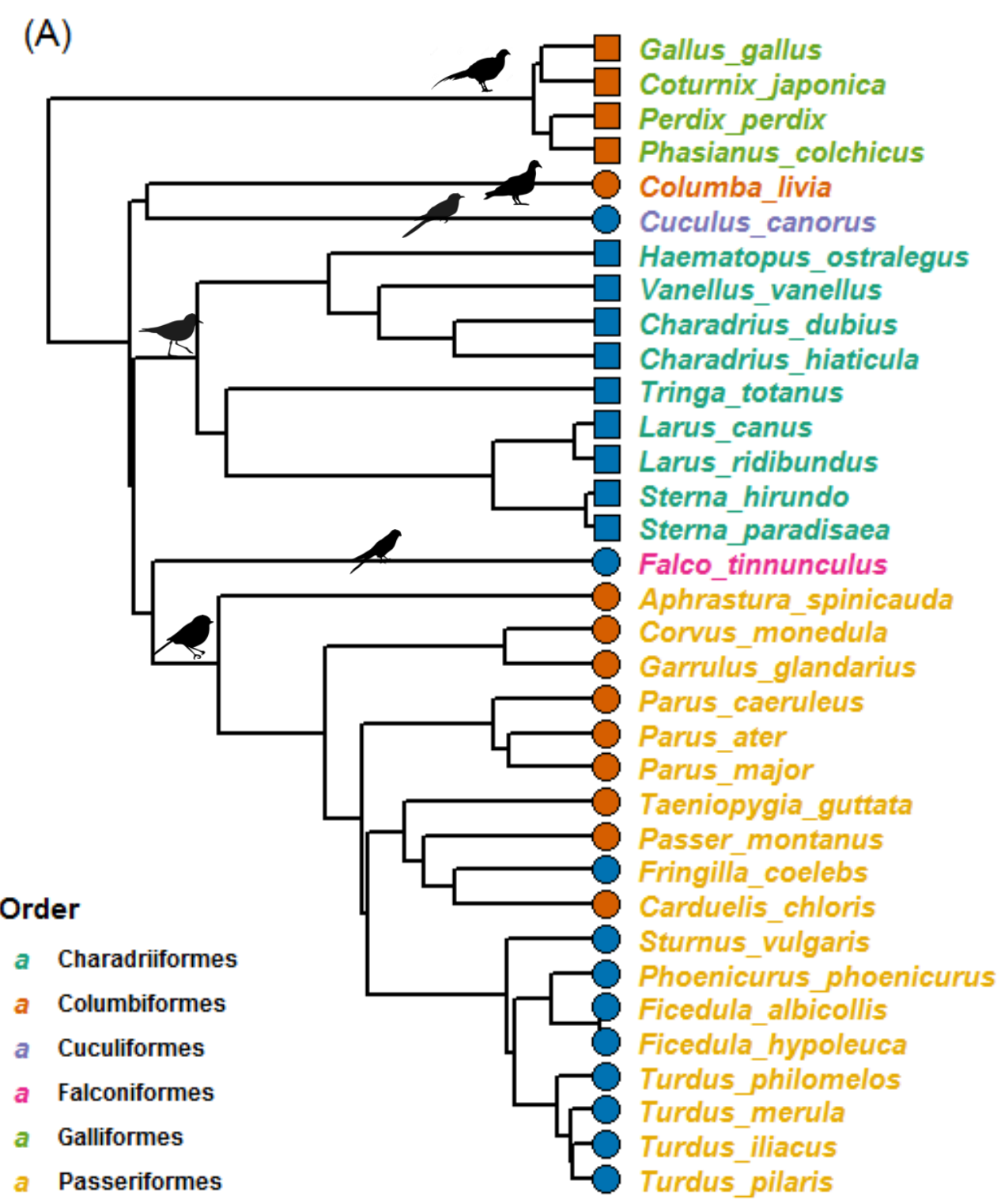

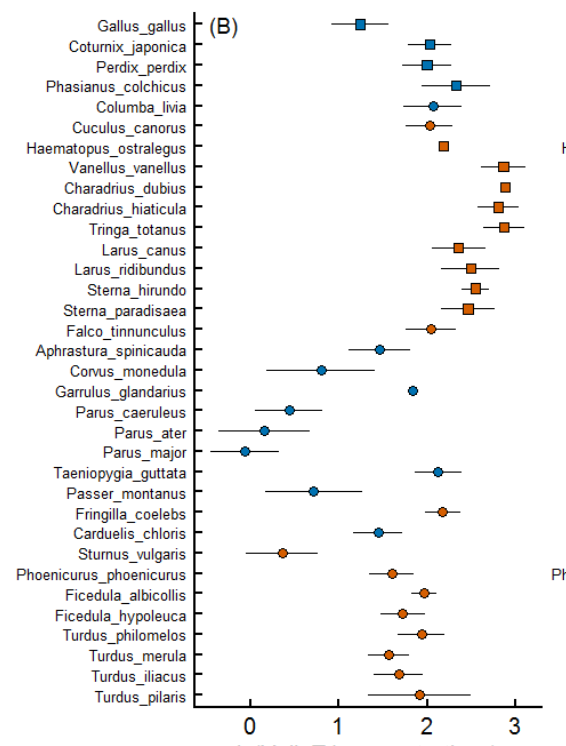

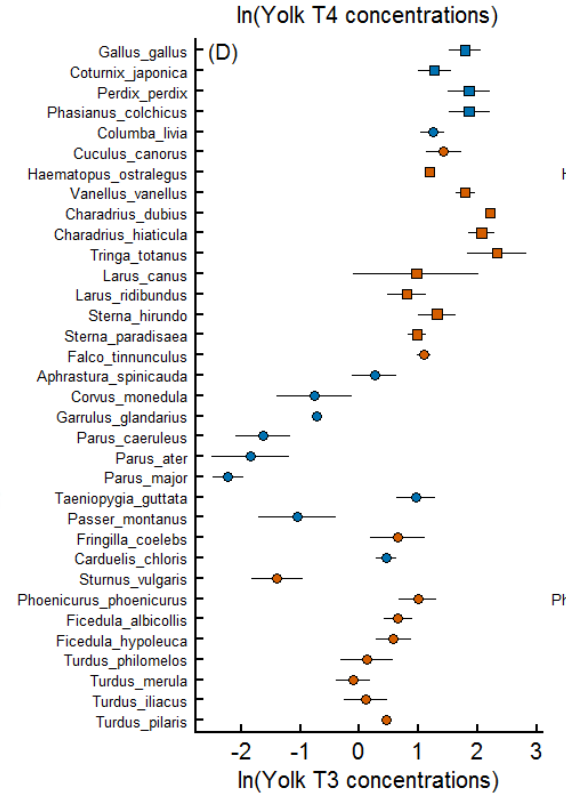


Figure 1. Phylogenetic tree, yolk TH concentrations and total contents of the avian species included in this study. The phylogenetic tree (A) is one possible tree derived from the Hackett backbone (see text). Different colours in species names represent different orders they belong to. Yolk TH concentrations (B, D) and total contents (C, E) exhibited substantial inter-specific variation (mean \pm SD, see Table S1 for exact values and sample sizes). Red symbols represent resident species, while blue symbols migratory species. Circles represent altricial species, and squares precocial species. 


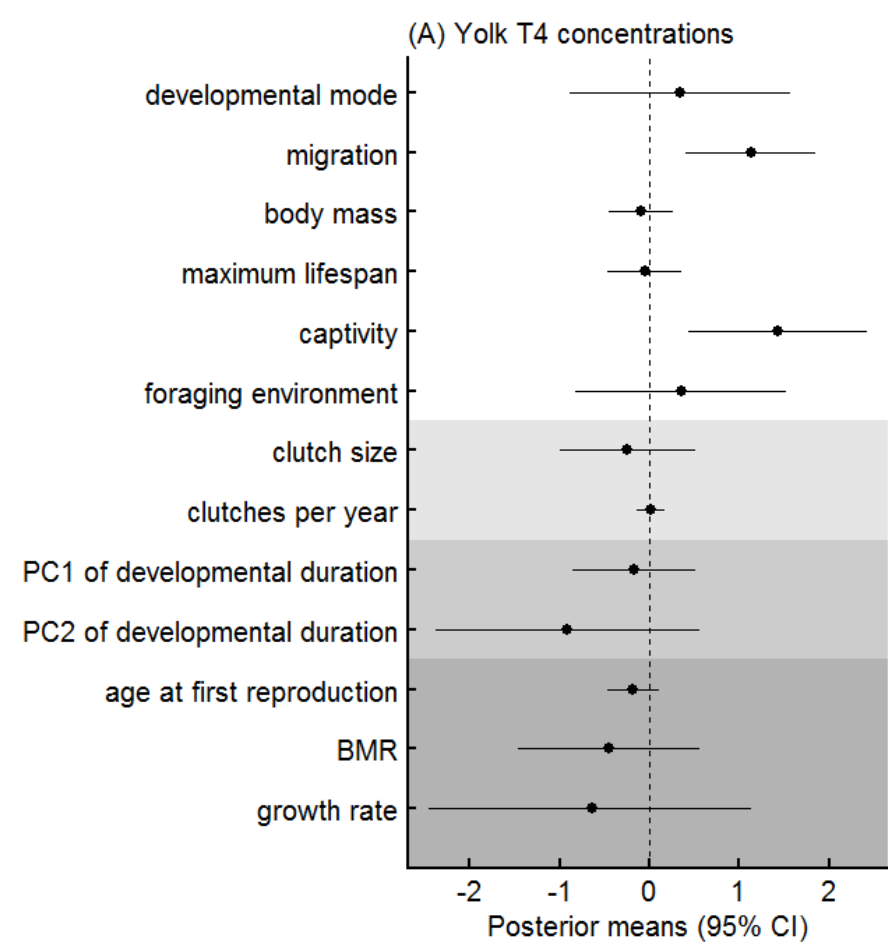

(C) Yolk T3 concentrations

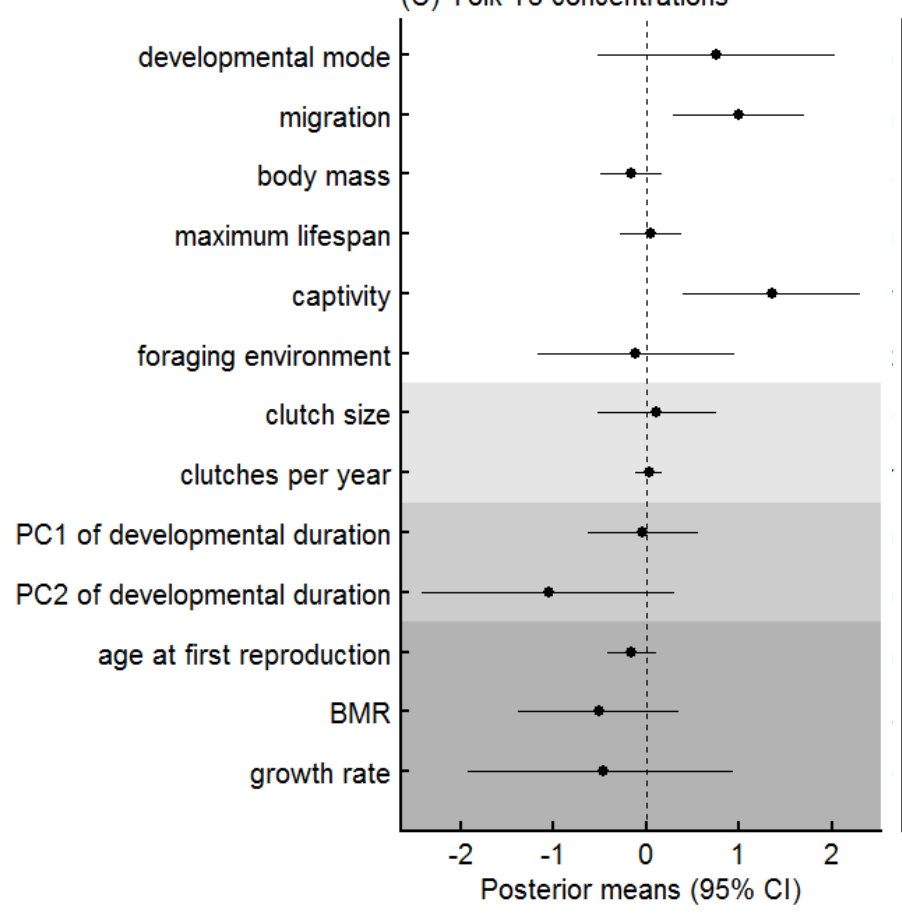

(B) Yolk T4 contents

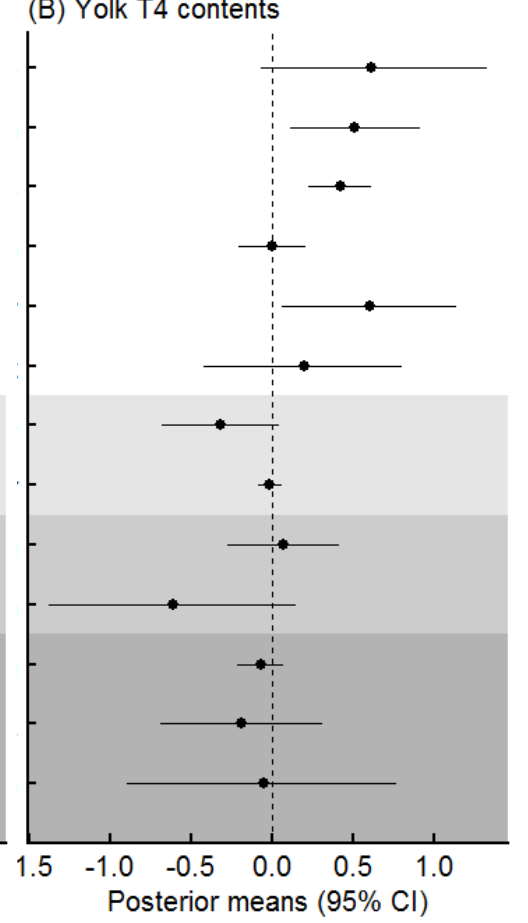

(D) Yolk T3 contents

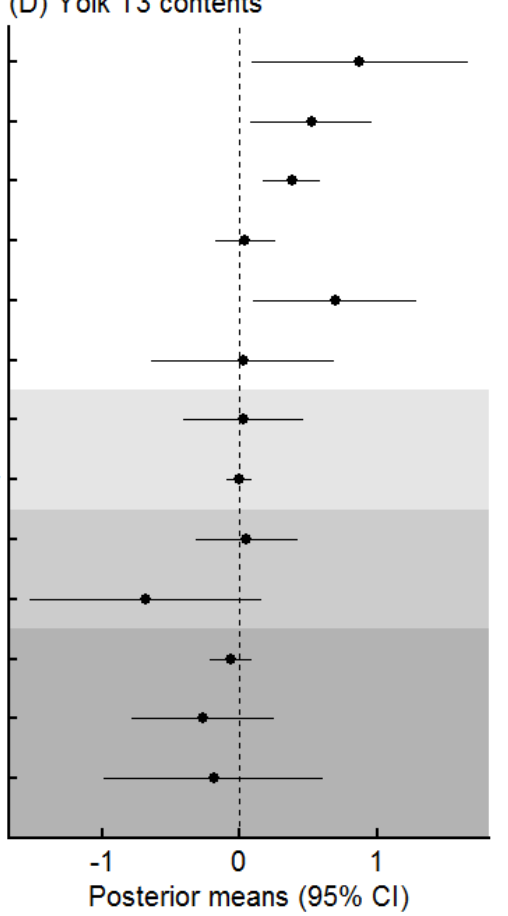

Figure 2. Posterior means ( $\pm 95 \%$ credible intervals) between yolk THs and all life-history variables

tested in this study. White area presents the estimates from model set 1 (see text and Table 1). The 
bioRxiv preprint doi: https://doi.org/10.1101/775981; this version posted November 7, 2019. The copyright holder for this preprint (which was not certified by peer review) is the author/funder, who has granted bioRxiv a license to display the preprint in perpetuity. It is made available under aCC-BY-NC-ND 4.0 International license.

541 estimate represents the difference in migratory species from resident species. For captivity, the

542 estimate represents the difference in captive species from wild species. For foraging environment,

543 the estimate represents the difference in marine-bound species from terrestrial species. 

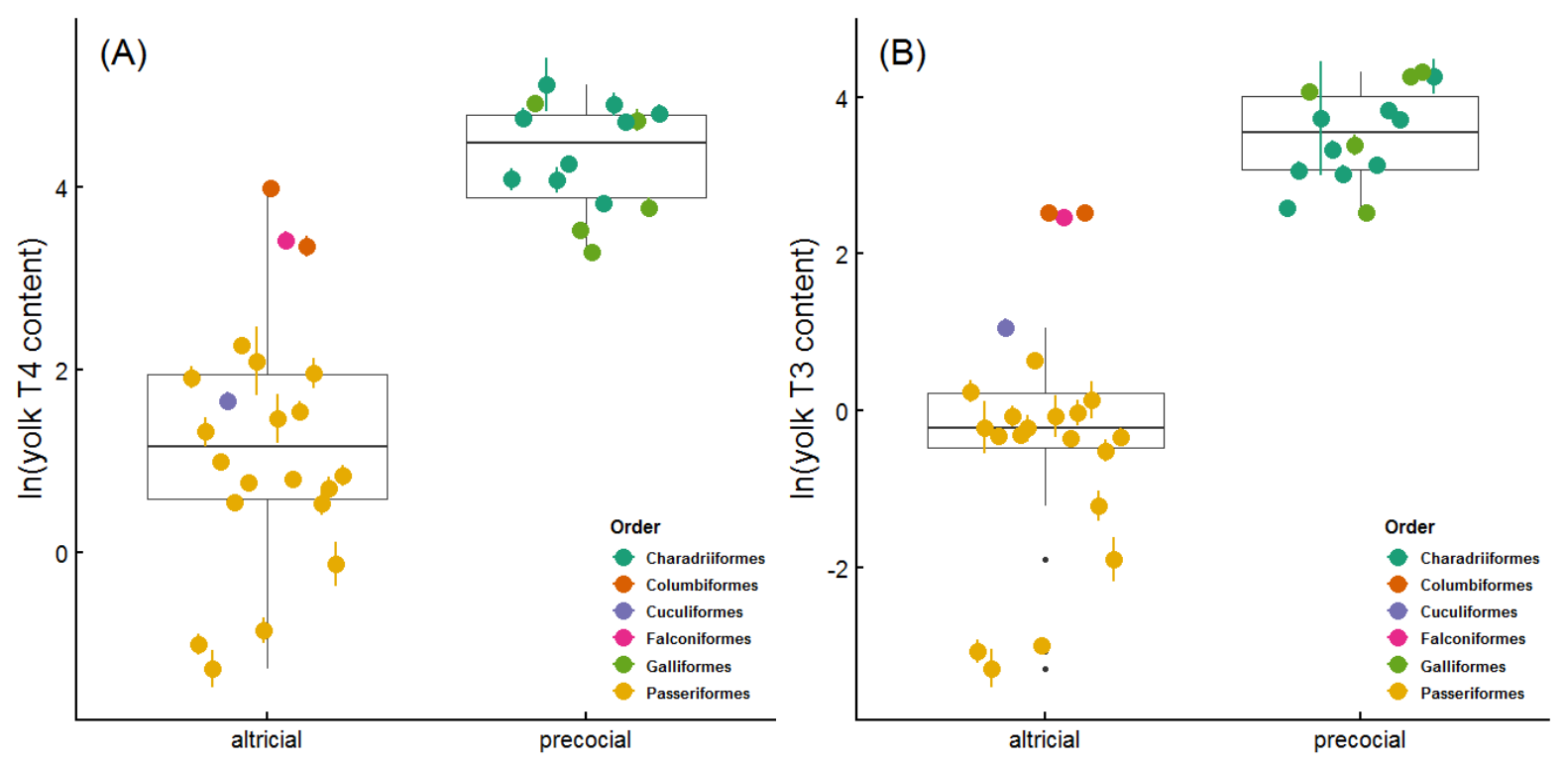

545 Figure 3. Boxplots and species-specific averages of yolk T4 (A) and T3 (B) contents (ng/yolk, In-

546 transformed raw data) according to developmental mode. Boxplots represent the median (the

547 middle line) and the first and the third quartiles (the box), and the whiskers extend to 1.5 times of

548 the interquartile range. Colored dots represent species-specific means $( \pm \mathrm{SE})$. 

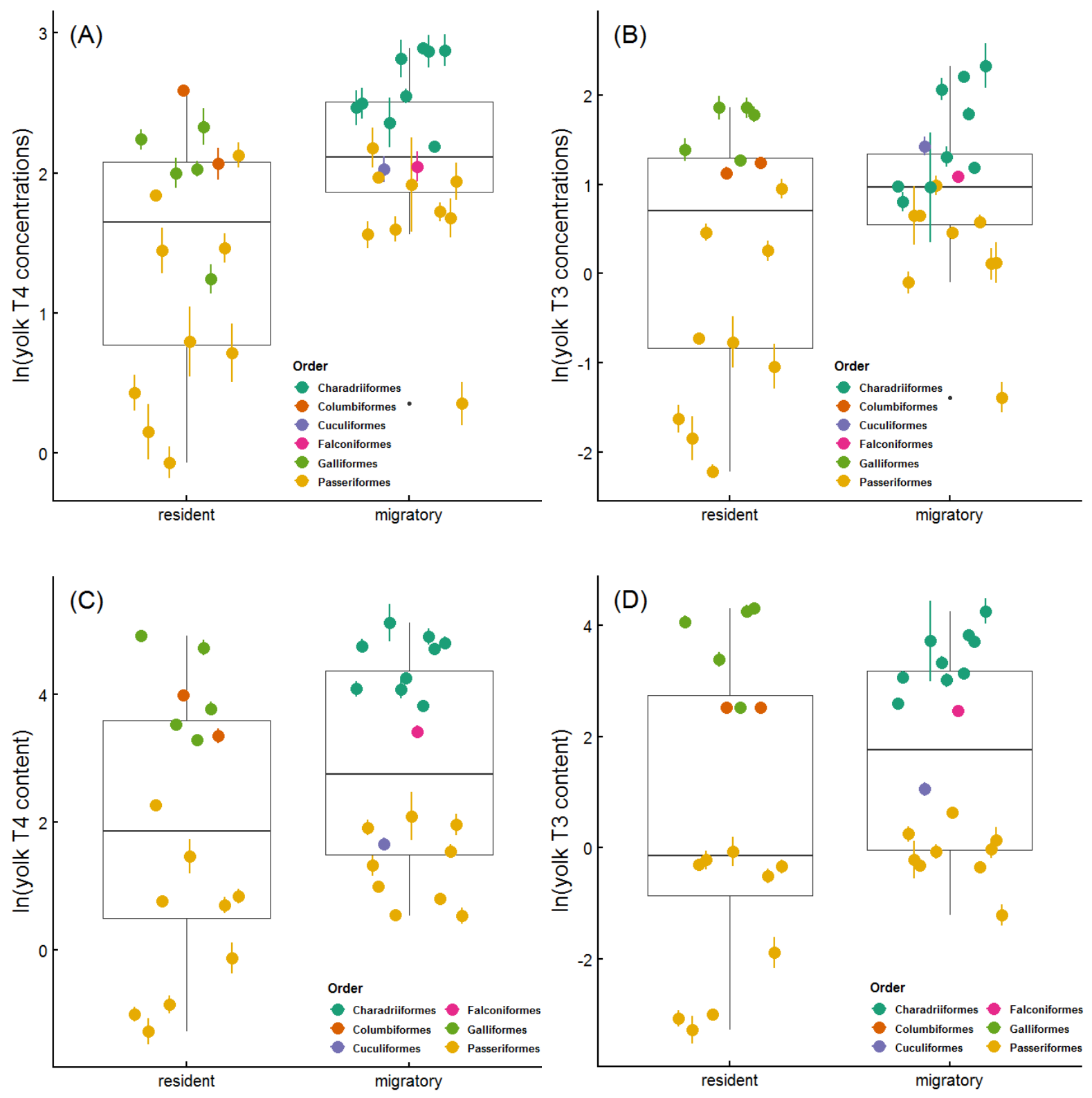

549

Figure 4. Boxplots and species-specific averages of yolk TH concentrations (A, B, pg/mg, In-

551 transformed raw data) and total contents (C, D, ng/yolk, In-transformed raw data) between

552 migratory and resident species. Boxplots represent the median (the middle line) and the first and the

553 third quartiles (the box), and the whiskers extend to 1.5 times of the interquartile range. Colored

554 dots represent species-specific means $( \pm S E)$. 

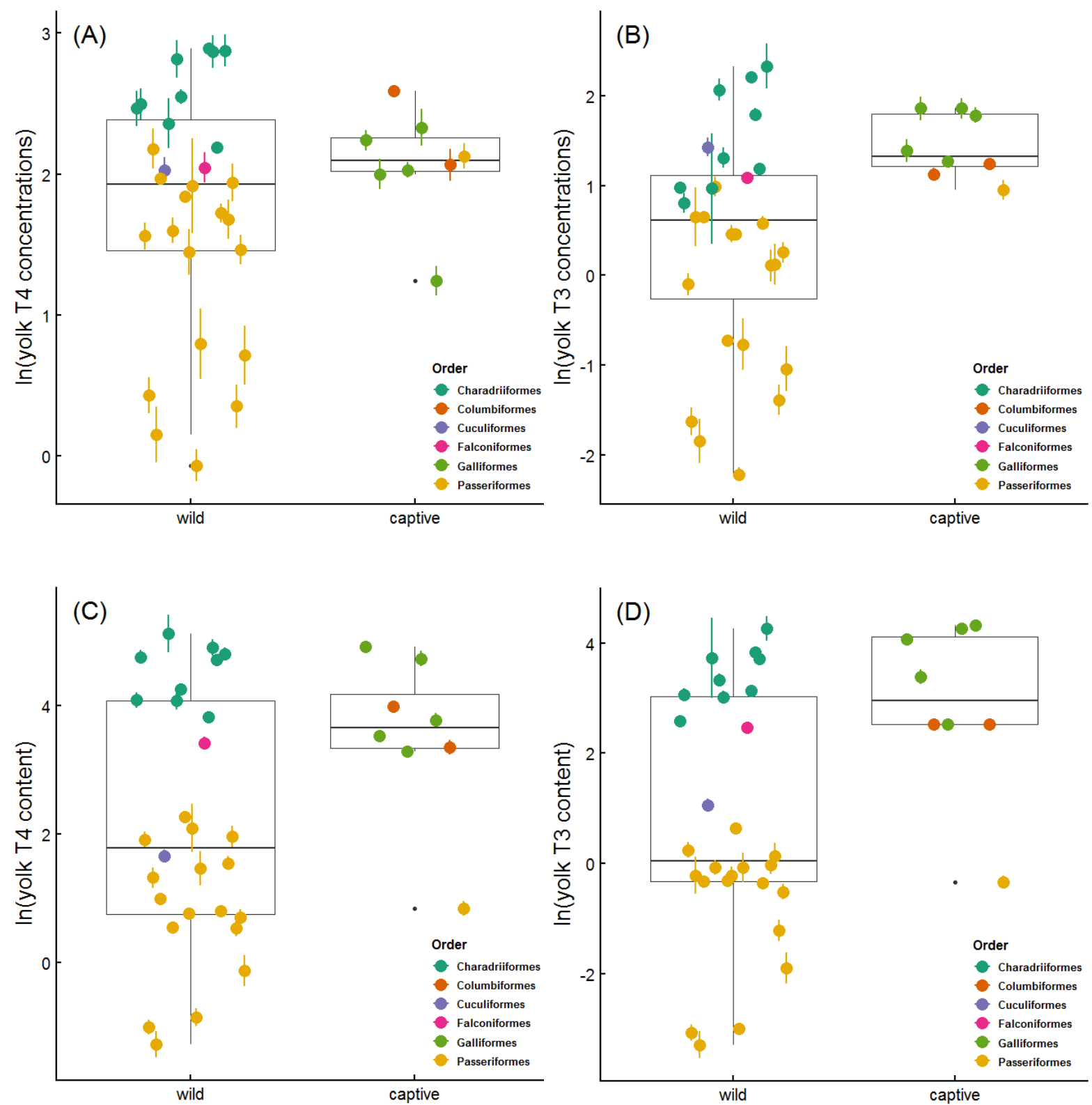

556 Figure 5. Boxplots of yolk TH concentrations (A, B, pg/mg, In-transformed raw data) and total

557 contents (ng/yolk, In-transformed raw data) in between captive and wild species. Boxplots represent

558 the median (the middle line) and the first and the third quartiles (the box), and the whiskers extend to 1.5 times of the interquartile range. Colored dots represent species-specific means ( $\pm \mathrm{SE})$. 
Angelier, F., Parenteau, C., Ruault, S., Angelier, N. (2016). Endocrine consequences of an acute stress under different thermal conditions: a study of corticosterone, prolactin, and thyroid hormones in the pigeon (Columba livia). Comp. Biochem. Physiol. A, 196, 38-45.

Auer, S.K., Dick, C.A., Metcalfe, N.B., Reznick, D.N. (2018). Metabolic rate evolves rapidly and in parallel with the pace of life history. Nat. Commun., 9, 14 status in UK pregnant women on cognitive outcomes in their children: results from the Avon Longitudinal Study of Parents and Children (ALSPAC). Lancet, 382, 331-337. not correlated with Dio2 expression in a wild male songbird, Sturnus vulgaris, exposed to natural changes in photoperiod. Endocrinology, 154, 1813-1819. Bizhanova, A., Kopp, P. (2009). Minireview: the sodium-iodide symporter NIS and pendrin in iodide homeostasis of the thyroid. Endocrinology, 150, 1084-1090. Boggs, A.S.P., Hamlin, H.J., Lowers, R.H., Guillette, L.J. Jr. (2011). Seasonal variation in plasma thyroid hormone concentration in coastal versus inland populations of juvenile American alligators (Alligator mississippiensis): influence of plasma iodide concentrations. Gen. Comp. Endocrinol., 174: 362-369. metabolism and the timing of reproduction in house sparrows Passer domesticus. J. Avian Biol., 34, 298-306.

Clements, J.F., Schulenberg, T.S., Iliff, M.J., Roberson, D., Fredericks, T.A., Sullivan, B.L. et al. (2018).

581 The eBird/Clements checklist of birds of the world: v2018. Downloaded from

582 http://www.birds.cornell.edu/clementschecklist/download/

583 Cortés, D.C.C., Langlois, V.S., Fernandino, J.I. (2014). Crossover of the hypothalamic pituitaryadrenal/interrenal-thyroid, and-gonadal axes in testicular development. Front. Endocrinol., 5, 139. 
Darras, V.M. (2019). The role of maternal thyroid hormones in avian embryonic development. Front.

Darras, V.M., Kotanen, S.P., Geris, K.L., Berghman, L.R., Kühn, E.R. (1996). Plasma thyroid hormone hormone. Front. Endocrinol., 4, 63.

Driscoll, C.A., Macdonald, D.W., O’Brien, S.J. (2009). From wild animals to domesticated pets, an evolutionary view of domestication. Proc. Natl. Acad. Sci. U.S.A., 106, 9971-9978.

Eckhoff, K.M., Maage, A. (1997). lodine content in fish and other food products from east Africa analyzed by ICP-MS. J. Food Compost. Anal., 10, 270-282. seabirds. Biol. Open, 2, 580-586. level phylogeny and morphological evolution of tyrant flycatchers, cotingas, manakins, and their allies (Aves: Tyrannida). Mol. Phylogenet. Evol., 40, 471-483. differ in wild and captive populations of the zebra finch. Anim. Behav., 85, 1329-1334. 
Hadfield, J.D. (2010). MCMC methods for multi-response generalized linear mixed models: the

Hadfield, J.D., Nakagawa, S. (2010). General quantitative genetic methods for comparative biology: Biol., 23, 494-508.

Hsu, B.-Y., Dijkstra, C., Darras, V.M., de Vries, B., Groothuis, T.G.G. (2016). Maternal adjustment of constraint: differential effects of food availability on maternal deposition of macro-nutrients, steroids and thyroid hormones in rock pigeon eggs. Ecol. Evol., 6, 397-411. livia). Gen. Comp. Endocrinol., 240, 174-181. elevated maternal thyroid hormones at no apparent oxidative cost during early postnatal period. $J$. Avian Biol., 50, e01919.

Hsu, B.-Y., Verhagen, I., Gienapp, P., Darras, V.M., Visser, M.E., Ruuskanen, S. (2019b). Between- and 632 within-individual variation of maternal thyroid hormone deposition in wild great tits (Parus major). Am. Nat., 194, E96-E108. Jetz, W., Freckleton, R.P., McKechnie, A.E. (2008). Environment, migratory tendency, phylogeny and basal metabolic rate in birds. PLOS ONE, 3, e3261. 
Jetz, W., Thomas, G.H., Joy, J.B., Hartmann, K., Mooers, A.O. (2012). The global diversity of birds in space and time. Nature, 491, 444-448. 5309-5324. Linnut. Turun Lintutieteellinen Yhdistys, Turku. (In Finnish) environment as a limiting factor for roe deer antler development. Biol. Trace Elem. Res., 139, 168176. experiments. Bioinformatics, 26, 966-968. method. Trends Ecol. Evol., 25, 713-721. McGlothin, J.W., Galloway, L.F. (2014). The contribution of maternal effects to selection response: an empirical test of competing models. Evolution, 68, 549-558.

657 McKechnie, A.E., Freckleton, R.P., Jetz, W. (2006). Phenotypic plasticity in the scaling of avian basal metabolic rate. Proc. R. Soc. B, 273, 931-937. 
McNabb, F.M.A. (2006). Avian thyroid development and adaptive plasticity. Gen. Comp. Endocrinol., $147,93-101$.

McNabb, F.M.A., Darras, V.M. (2015). Thyroids. In: Sturkie's Avian Physiology, $6^{\text {th }}$ edition. (ed. C.G.

Medici, M., Timmermans, S., Visser, W., de Muinck Keizer-Schrama, S.M.P.F., Jaddoe, V.W.W.,

Moreno, J., Merino, S., Vásquez, R.A., Armesto, J.J. (2005). Breeding biology of the thorn-tailed rayadito (Furnariidae) in south-temperate rainforests of Chile. Condor, 107, 69-77. York, NY. 355-382. life-history database to perform comparative analyses with birds, mammals, and reptiles. Ecology, 96, 3109.

Nakagawa, S., Schielzeth, H. (2010). Repeatability for Gaussian and non-Gaussian data: a practical guide for biologists. Biol. Rev., 85, 935-956. pars tuberalis triggers photoperiodic response. Nature, 452, 317-323. 
Pap, P.L., Vágási, C.I., Vincze, O., Osváth, G., Veres-Szászka, J., Czirják, G.Á. (2015). Physiological pace

Gambel's white-crowned sparrows: role of thyroxine and triiodothyronine. Horm. Behav., 84, 50-56. for MCMC. R News, 6, 7-11. concentrations in the chicken egg and in the embryo before and after the onset of thyroid function.

Price, E.R., Dzialowski, E.M. (2018). Development of endothermy in birds: patterns and mechanisms.

Revell, L.J. (2009). Size-correction and principal components for interspecific comparative studies.

710 Revell, L.J. (2012). phytools: an R package for phylogenetic comparative biology (and other things). 
Rubolini, D., Liker, A., Garamszegi, L.Z., Møller, A.P., Saino, N. (2015). Using the BirdTree.org website Heritable variation in maternally derived yolk androgens, thyroid hormones, and immune factors. Heredity, 117, 184-190.

Starck, J.M., Ricklefs, R.E. (1998a). Patterns of development: the altricial-precocial spectrum. In: Press, New York, NY, pp. 381-415. 

ageing genomic resources: Integrated databases and tools for the biology and genetics of ageing. Nucleic Acids Res., 41, D1027-D1033. baseline blood glucose concentration co-evolves with life-history traits in songbirds. Funct. Ecol., 33, 239-249.

Trumpff, C., de Schepper, J., Tafforeau, J., van Oyen, H., Vanderfaeillie, J., Vandervijvere, S. (2013). Mild iodine deficiency in pregnancy in Europe and its consequences for cognitive and psychomotor development of children: a review. J. Trace Elem. Med. Biol., 27, 174-183.

Vágási, C.I., Vincze, O., Pătraş, L., Osváth, G., Pénzes, J., Haussmann, M.F. et al. (2019). Longevity and life history coevolve with oxidative stress in birds. Funct. Ecol., 33, 152-161. van Herck, S.L.J., Geysens, S., Delbaere, J., Tylzanowski, P. Darras, V.M. (2012). Expression profile and thyroid hormone responsiveness of transporters and deiodinases in early embryonic chicken brain development. Mol. Cell. Endocrinol., 349, 289-297. thermoregulatory consequences of molting on metabolic rate in a northerly wintering shorebird. Physiol. Biochem. Zool., 82, 129-142. of oxidative stress and neurodegeneration. Oxid. Med. Cell. Longev. 2013, 218145. species. PLOS ONE, 8, e56229. 
bioRxiv preprint doi: https://doi.org/10.1101/775981; this version posted November 7, 2019. The copyright holder for this preprint (which

was not certified by peer review) is the author/funder, who has granted bioRxiv a license to display the preprint in perpetuity. It is made available under aCC-BY-NC-ND 4.0 International license.

762 Wikelski, M., Spinney, L., Schelsky, W., Scheuerlein, A., Gwinner, E. (2003). Slow pace of life in

763 tropical sedentary birds: a common-garden experiment on four stonechat populations from different

764 latitudes. Proc. $R$ Soc. Lond. B, 270, 2383-2388.

765 Zhao, L., Gao, L., Yang, W., Xu, X., Wang, W., Liang, W. et al. (2017). Do migrant and resident species

766 differ in the timing of increases in reproductive and thyroid hormone secretion and body mass? A

767 case study in the comparison of pre-breeding physiology rhythms in the Eurasian skylark and Asian

768 short-toed lark. Avian Res., 8, 10. 